\title{
Assessment of Neonatal Abstinence Syndrome Surveillance - Pennsylvania, 2019
}

\author{
Kathleen H. Krause, $\mathrm{PhD}^{1,2}$; Joann F. Gruber, $\mathrm{PhD}^{1,3}$; Elizabeth C. Ailes, $\mathrm{PhD}^{2}$; Kayla N. Anderson, $\mathrm{PhD}^{2}$; Victoria L. Fields, DVM ${ }^{1,2}$; \\ Kimberlea Hauser, MBA ${ }^{3}$; Callie L. Howells, MS³; Allison Longenberger, PhD 3 ; Nancy McClung, PhD ${ }^{1,4}$; Lisa P. Oakley, PhD ${ }^{1,5}$; \\ Jennita Reefhuis, $\mathrm{PhD}^{2}$; Margaret A. Honein, $\mathrm{PhD}^{2}$; Sharon M. Watkins, $\mathrm{PhD}^{3}$
}

The incidence of neonatal abstinence syndrome (NAS), a withdrawal syndrome associated with prenatal opioid or other substance exposure (1), has increased as part of the U.S. opioid crisis (2). No national NAS surveillance system exists (3), and data about the accuracy of state-based surveillance are limited (4,5). In February 2018, the Pennsylvania Department of Health began surveillance for opioid-related NAS in birthing facilities and pediatric hospitals* (6). In March 2019, CDC helped the Pennsylvania Department of Health assess the accuracy of this reporting system at five Pennsylvania hospitals. Medical records of 445 infants who possibly had NAS were abstracted; these infants had either been reported by hospital providers as having NAS or assigned an International Classification of Diseases, Tenth Revision, Clinical Modification (ICD-10-CM) hospital discharge code potentially related to NAS. ${ }^{\dagger}$ Among these 445 infants, 241 were confirmed as having NAS. Pennsylvania's NAS surveillance identified 191 (sensitivity $=79 \%$ ) of the confirmed cases. The proportion of infants with confirmed NAS who were assigned the ICD-10-CM code for neonatal withdrawal symptoms from maternal use of drugs of addiction (P96.1) was similar among infants reported to surveillance $(71 \%)$ and those who were not $(78 \% ; p=0.30)$. Infants with confirmed NAS who were not assigned code P96.1 typically had less severe signs and symptoms. Accurate NAS surveillance, which is necessary to monitor changes and regional differences in incidence and assist with planning for needed services, includes and is strengthened by a combination of diagnosis code assessment and focused medical record review.

Five Pennsylvania hospitals were selected to represent various sizes, geographic regions, and anticipated NAS incidence. A

\footnotetext{
*As described in the Neonatal Abstinence Syndrome: 2018 Report released by the Pennsylvania Bureau of Epidemiology, the Pennsylvania NAS surveillance system was established through an emergency declaration that made NAS a reportable condition throughout the state. The Pennsylvania NAS surveillance case definition required health care providers at birthing facilities and pediatric hospitals to report infants born on or after January 10, 2018, to residents of Pennsylvania who received a diagnosis of NAS (based on prenatal exposure to opiate drugs anytime during pregnancy and the presence of at least one symptom of withdrawal) during the neonatal period (birth through 28 days of life). Reports were submitted through a web-based system.

$\dagger$ Included the following ICD-10 codes available as of October, 2018: F11.x; T40.0x-T40.4x, T40.6x, T50.7x; P96.1; P04.1x, P04.49, P04.89, P04.9; P04.2, P04.3, P04.41, P04.42, Q86.0; P90, R56.xx; P81.8, P81.9; R25.1, R25.8, R25.9; P94.1, P94.8, P94.9.
}

broad NAS case definition was used to identify infants who possibly had NAS under the a priori assumption that hospitals might not always assign an infant P96.1 or a clinical diagnosis of NAS, despite the presence of NAS symptoms. Infants who possibly had NAS were aged $<28$ days born during March 1August 31, 2018, and either reported to NAS surveillance or assigned a hospital discharge ICD-10-CM code indicative of prenatal substance exposure or NAS symptom. ${ }^{\S}$ Medical records of all infants who possibly had NAS were reviewed for demographic and birth characteristics, prenatal opioid and other substance exposure, infant and maternal toxicology results and NAS symptoms and treatment information. Infants were considered to have confirmed NAS if all of the following criteria were documented in the infant medical record: 1) at least one NAS symptom; 2) maternal history or toxicology results indicating prenatal opioid exposure; and 3) a clinical mention of NAS (i.e., NAS listed in the discharge diagnosis or problem list or use of a NAS scoring tool [e.g., Finnegan]). For infants with confirmed NAS, maternal prenatal and delivery records were abstracted to gather additional data on prenatal opioid or other substance exposure.

Sensitivity and positive predictive value (PPV) of the Pennsylvania NAS surveillance system were calculated, with corresponding $95 \%$ confidence intervals (CIs) estimated using an exact binomial distribution. Descriptive analyses compared infants with confirmed NAS by reporting status and by presence of ICD-10-CM code P96.1. Categorical variables were compared using chi-squared tests (or Fisher's exact tests for cell counts $<5$ ); continuous variables were compared using negative binomial regression. Statistical significance was assessed at $a=0.05$. All analyses were conducted using SAS (version 9.4; SAS Institute). This activity was reviewed by CDC and was conducted consistent with applicable federal law and CDC policy.

\footnotetext{
$\$$ Signs and symptoms include tremors, breathing problems, blotchy skin, diarrhea, crying, fever, fussiness, gagging or retching, hiccups, hyperactive or exaggerated Moro reflex, frequent yawning, overactive reflexes, poor feeding, salivation, seizures, skin abrasions or excoriation, slow weight gain, sneezing, stuffy nose, suckling issues, sweating, vomiting, increased muscle tone, trouble sleeping, and any other symptom attributed to NAS by a clinician.

95 C.F.R. part 46.102(I)(2), 21 C.F.R. part 56; 42 U.S.C. Sect. 241(d); 5 U.S.C. Sect. 552a; 44 U.S.C. Sect. 3501 et seq.
} 
Overall, 445 infants who possibly had NAS were identified: 192 were reported to surveillance and 253 identified through diagnosis codes alone (Figure). Medical record review identified 241 infants with confirmed NAS, 191 of whom were reported to surveillance (sensitivity $=79 \%$ [191 of $241 ; 95 \% \mathrm{CI}=74 \%-84 \%]$; PPV $=99 \%$ [191 of 192; $95 \% \mathrm{CI}=97 \%-100 \%])$. Among the 241 infants with confirmed NAS, those reported to surveillance were significantly more likely than were those not reported to have documentation of neonatal (69\% versus 50\%) or maternal $(55 \%$ versus $30 \%)$ toxicology evidence of prenatal opioid exposure in the infant record, maternal history of prenatal opioid exposure in the maternal record ( $98 \%$ versus $90 \%$ ), and prenatal exposure to cannabis (30\% versus $10 \%$ ) in the infant or maternal record (Table 1 ). Notably, $71 \%$ of infants reported to surveillance were assigned ICD-10-CM code P96.1, which was not significantly different from infants not reported (78\%).

Among infants with confirmed NAS, type and source of opioid exposure were similar in those who were and were not assigned P96.1 (Table 2). However, infants assigned P96.1 were more likely than were those not assigned P96.1 to have mothers enrolled in Medicaid (95\% versus $88 \%$ ), significantly longer lengths of stay ( 14 versus 9 days), older ages at first NAS score ( 2 versus 1 days), higher first NAS scores (4 versus 2), older ages at peak NAS score (5 versus 3 days), higher peak NAS scores (11 versus 9), more NAS symptoms (12 versus 9), more frequent pharmacologic treatment (61\% versus 3\%), and greater prenatal exposure to gabapentin in the infant or maternal record (12\% versus $1 \%$ ). Infants not assigned P96.1 were significantly more likely to be assigned ICD-10-CM code P04.49, "Newborn suspected to be affected by maternal use of other drugs of addiction" (60\% versus $23 \%$ ).

\section{Discussion}

Based on medical record review at five hospitals, Pennsylvania's NAS surveillance system had a PPV of $99 \%$ and sensitivity of $79 \%$. Accurate NAS surveillance is necessary to monitor temporal and geographic changes in NAS incidence and to plan for needed services. Findings from this evaluation might inform NAS surveillance efforts in other states. First, ICD-10-CM code P96.1 was assigned to $71 \%$ of infants reported to Pennsylvania's NAS surveillance system, demonstrating the utility of using this code to efficiently identify NAS cases. However, $78 \%$ of infants not reported to the system were also assigned P96.1. Infants who are assigned P96.1 meet the Council of State and Territorial Epidemiologists (CSTE) 2019 Tier 2 confirmed NAS case definition (1), which was released after this investigation. CSTE's standardized definition might help clarify which infants should be reported for future surveillance efforts. Previous studies have found that use of P96.1
FIGURE. Identification of infants with confirmed neonatal abstinence syndrome (NAS) through medical record review of those reported to NAS surveillance and those identified by diagnosis codes selected hospitals, Pennsylvania, 2018

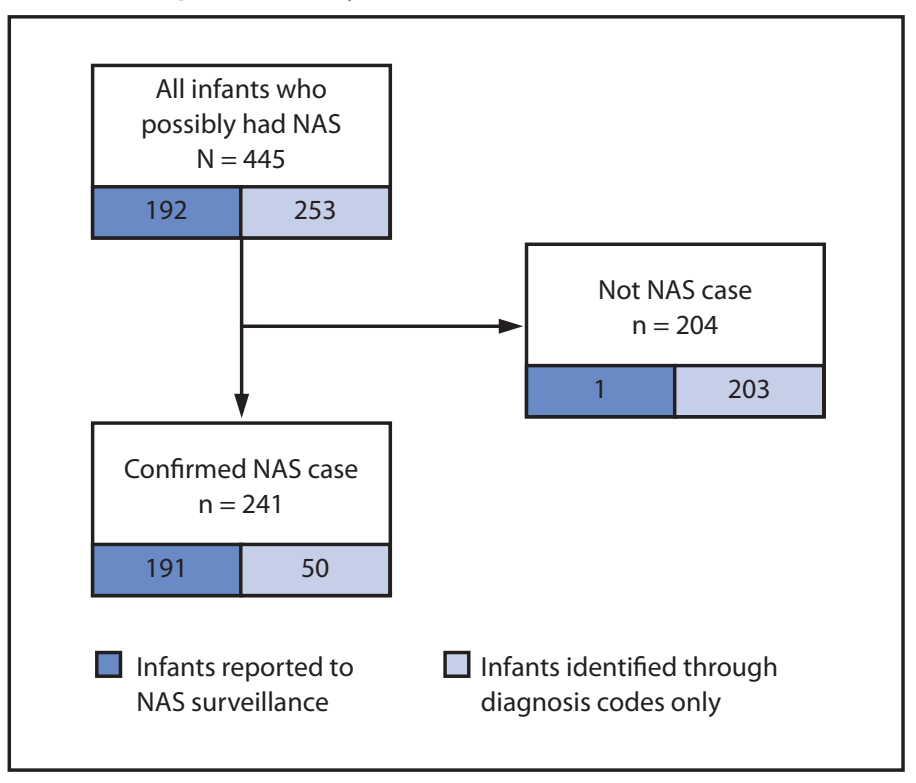

\section{Summary}

What is already known about this topic?

Neonatal abstinence syndrome (NAS) has increased as part of the U.S. opioid crisis, but no national NAS surveillance system exists, and data about the accuracy of state-based surveillance are limited.

What is added by this report?

Among infants with confirmed NAS at five Pennsylvania hospitals, ICD-10-CM code P96.1 was assigned to $71 \%$ of those who were reported to the NAS surveillance system and $78 \%$ of those who were not reported to surveillance.

What are the implications for public health practice?

Accurate NAS surveillance, which is necessary to monitor changes and regional differences in incidence and assist with planning for needed services, includes a combination of diagnosis code assessment and focused medical record review.

to identify infants with NAS can yield high PPV $(4,7,8)$, and a combination of P96.1 or P04.49 improves sensitivity but decreases PPV (5). Second, in this investigation, infants with more severe signs and symptoms of NAS were more likely to be assigned P96.1. A recent review of surveillance practices highlighted the variability of NAS case definitions and use of ICD-10-CM codes across jurisdictions (9). Consistency in coding of infants with NAS could assist future surveillance efforts. Third, infants with toxicology evidence of prenatal opioid exposure were more likely to be reported to surveillance, but toxicology evidence was also frequently found among unreported cases. CSTE's Tier 1 NAS confirmed case 
TABLE 1. Characteristics of infants with confirmed neonatal abstinence syndrome (NAS) based on medical record review (N $=241$ ) who were reported and not reported to surveillance - selected hospitals, Pennsylvania, 2018

\begin{tabular}{|c|c|c|c|c|}
\hline \multirow[b]{2}{*}{ Characteristic } & \multicolumn{3}{|c|}{ No.* (\%) or mean (range) } & \multirow[b]{2}{*}{$p$-value ${ }^{\dagger}$} \\
\hline & $\begin{array}{l}\text { All infants with NAS } \\
\qquad(\mathrm{N}=241)\end{array}$ & $\begin{array}{l}\text { Infants reported to } \\
\text { surveillance } \\
(\mathrm{N}=191)\end{array}$ & $\begin{array}{l}\text { Infants not reported to } \\
\text { surveillance, identified } \\
\text { through diagnosis codes only } \\
\qquad(\mathrm{N}=50)\end{array}$ & \\
\hline \multicolumn{5}{|l|}{ Maternal race } \\
\hline White & $211(91)$ & $171(92)$ & $40(83)$ & \\
\hline Other ${ }^{\S}$ & $22(9)$ & $14(8)$ & $8(17)$ & 0.055 \\
\hline \multicolumn{5}{|l|}{ Maternal ethnicity } \\
\hline Hispanic or Latina & $2(>1)$ & $1(1)$ & $1(2)$ & \\
\hline Not Hispanic or Latina & $223(99)$ & $179(99)$ & $44(98)$ & 0.361 \\
\hline \multicolumn{5}{|c|}{ Source of payment in maternal record } \\
\hline Medicaid & $216(93)$ & $174(94)$ & $42(91)$ & 0.530 \\
\hline Private/Other & $16(7)$ & $12(6)$ & $4(9)$ & \\
\hline Maternal age, yrs & $234^{\Uparrow} ; 29(18-43)$ & $184^{\uparrow} ; 29(18-43)$ & $50 \% ; 30(22-40)$ & 0.112 \\
\hline \multicolumn{5}{|l|}{ Infant sex } \\
\hline Male & $118(49)$ & $97(51)$ & $21(42)$ & \\
\hline Female & $123(51)$ & $94(49)$ & $29(58)$ & 0.269 \\
\hline Gestational age, wks & $235^{\oplus ;} ; 38(32-42)$ & $187^{\uparrow} ; 38(32-41)$ & $48^{\Uparrow} ; 37(32-42)$ & 0.417 \\
\hline \multicolumn{5}{|l|}{ Type of hospitalization } \\
\hline Birth hospitalization & $221(92)$ & $178(93)$ & $43(88)$ & \\
\hline Other type of admission & $19(8)$ & $13(7)$ & $6(12)$ & 0.208 \\
\hline Length of stay, days & 240 \%; $13(1-68)$ & 190 ; $13(2-68)$ & $50 \% ; 12(1-47)$ & 0.596 \\
\hline \multicolumn{5}{|l|}{ NAS scores } \\
\hline Age at first NAS score, days & 234ף; 1 (0-19) & $186^{\text {ๆ; }} 1$ (0-17) & 48 ; 2 (0-19) & 0.163 \\
\hline First NAS score ${ }^{* *}$ & 239 ; 3 (0-19) & $190 \% ; 3(0-14)$ & $49^{\oplus} ; 4$ (0-19) & 0.063 \\
\hline Age at highest NAS score, days & $2300^{1 ;} ;(0-32)$ & $182^{n} ; 5(0-32)$ & $48 \% ; 4(1-21)$ & 0.275 \\
\hline Highest NAS score** & 238 ; $10(2-21)$ & $1899^{\prime} ; 10(2-21)$ & 49 ; $; 10(2-19)$ & 0.659 \\
\hline \multicolumn{5}{|l|}{ Symptoms } \\
\hline Total number of symptoms ${ }^{\dagger \dagger}$ & 240 ; $11(1-17)$ & 191 甲; $12(1-17)$ & 49 \% $11(1-17)$ & 0.147 \\
\hline \multicolumn{5}{|c|}{ Evidence of prenatal opioid exposure in the infant record ${ }^{\S \S}$} \\
\hline Neonatal toxicology evidence & $157(65)$ & $132(69)$ & $25(50)$ & 0.012 \\
\hline Maternal toxicology evidence & $120(50)$ & $105(55)$ & $15(30)$ & 0.002 \\
\hline Maternal history & $225(93)$ & $178(93)$ & $47(94)$ & 1.000 \\
\hline \multicolumn{5}{|c|}{ Evidence of prenatal opioid exposure in the maternal prenatal or delivery record $\S^{\S}$} \\
\hline Maternal toxicology evidence & $56(23)$ & $44(23)$ & $12(24)$ & 0.886 \\
\hline Maternal history & $233(97)$ & $188(98)$ & $45(90)$ & 0.011 \\
\hline \multicolumn{5}{|l|}{ Type of opioid exposure ๆף $^{\text {T. }}$} \\
\hline Buprenorphine & $160(66)$ & $125(65)$ & $35(70)$ & 0.544 \\
\hline Methadone & $68(28)$ & $58(30)$ & $10(20)$ & 0.147 \\
\hline Opiates, unspecified & $69(29)$ & $57(30)$ & $12(24)$ & 0.416 \\
\hline Heroin & $40(17)$ & $35(18)$ & $5(10)$ & 0.159 \\
\hline Oxycodone & $30(12)$ & $22(12)$ & $8(16)$ & 0.393 \\
\hline Other opioids & $17(7)$ & $12(6)$ & $5(10)$ & 0.361 \\
\hline
\end{tabular}

See table footnotes on the next page.

definition requires, in part, that infants have neonatal laboratory evidence of exposure (1); therefore, information on all infants with toxicologic evidence of exposure might warrant review when conducting NAS surveillance.

Although using P96.1 to trigger case review could have improved reporting to surveillance because it would have identified $78 \%$ of unreported NAS cases, using P96.1 as the sole criterion for reporting would have missed $29 \%$ of all infants reported with NAS. Medical record review was needed to identify infants with NAS who were less likely to have toxicology evidence of exposure (among infants not reported) and more likely to have less severe signs and symptoms of NAS (among infants not assigned P96.1). Therefore, these data suggest that using both diagnosis code assessment and focused medical record review as case-finding methods, though the latter might be labor intensive, would most accurately identify infants with NAS. Notably, in this investigation, this strategy relied on reviewing medical records of a selected group of infants with diagnosis codes indicative of prenatal substance exposure or a NAS symptom, and not only NAS diagnosis codes. Additional work is needed to identify the optimal subset of codes to identify possible infants with NAS $(5,7,8)$. 
TABLE 1. (Continued) Characteristics of infants with confirmed neonatal abstinence syndrome (NAS) based on medical record review ( $\mathrm{N}=241$ ) who were reported and not reported to surveillance — selected hospitals, Pennsylvania, 2018

\begin{tabular}{|c|c|c|c|c|}
\hline \multirow[b]{2}{*}{ Characteristic } & \multicolumn{3}{|c|}{ No.* (\%) or mean (range) } & \multirow[b]{2}{*}{ p-value } \\
\hline & $\begin{array}{l}\text { All infants with NAS } \\
\qquad(N=241)\end{array}$ & $\begin{array}{l}\text { Infants reported to } \\
\text { surveillance } \\
(\mathrm{N}=191)\end{array}$ & $\begin{array}{l}\text { Infants not reported to } \\
\text { surveillance, identified } \\
\text { through diagnosis codes only } \\
\qquad(\mathrm{N}=50)\end{array}$ & \\
\hline \multicolumn{5}{|l|}{ Type of other exposure ${ }^{t+t}$} \\
\hline Tobacco & $179(74)$ & $146(76)$ & $33(66)$ & 0.133 \\
\hline Cannabis & $63(26)$ & $58(30)$ & $5(10)$ & 0.004 \\
\hline Cocaine & $38(16)$ & $34(18)$ & $4(8)$ & 0.126 \\
\hline Antidepressants & $35(15)$ & $25(13)$ & $10(20)$ & 0.217 \\
\hline Benzodiazepines & $34(14)$ & $24(13)$ & $10(20)$ & 0.179 \\
\hline Amphetamine & $27(11)$ & $23(12)$ & $4(8)$ & 0.614 \\
\hline Gabapentin & $22(9)$ & $17(9)$ & $5(10)$ & 0.810 \\
\hline \multicolumn{5}{|l|}{ Infant receipt of pharmacologic treatment for NAS } \\
\hline Yes & $107(44)$ & $87(46)$ & $20(42)$ & \\
\hline No & $129(54)$ & $101(54)$ & $28(58)$ & 0.567 \\
\hline \multicolumn{5}{|l|}{ ICD-10-CM discharge diagnosis codes $\S^{\S \S}$} \\
\hline $\begin{array}{l}\text { P96.1, Neonatal withdrawal symptoms from } \\
\text { maternal use of drugs of addiction }\end{array}$ & $174(72)$ & $135(71)$ & $39(78)$ & 0.304 \\
\hline $\begin{array}{l}\text { P04.1, Newborn (suspected to be) affected by other } \\
\text { maternal medication ( } 2018 \text { edition code) }\end{array}$ & $8(3)$ & $5(3)$ & $3(6)$ & 0.368 \\
\hline P04.2, Newborn affected by maternal use of tobacco & $10(4)$ & $7(4)$ & $3(6)$ & 0.437 \\
\hline P04.3, Newborn affected by maternal use of alcohol & $1(0)$ & $0(-)$ & $1(2)$ & 0.207 \\
\hline $\begin{array}{l}\text { P04.41, Newborn affected by maternal } \\
\text { use of cocaine }\end{array}$ & $5(2)$ & $4(2)$ & $1(2)$ & 1.000 \\
\hline $\begin{array}{l}\text { P04.49, Newborn (suspected to be) affected by } \\
\text { maternal use of other drugs of addiction }\end{array}$ & $80(33)$ & $64(34)$ & $16(32)$ & 0.840 \\
\hline
\end{tabular}

\footnotetext{
Abbreviations: ICD-10-CM = International Classification of Diseases, Tenth Revision, Clinical Modification; NAS = neonatal abstinence syndrome.

* Frequencies might not sum to total because of missing values. When data are not available for all members of a cohort, $n$ is stated.

† P-values were calculated comparing infants reported to surveillance with infants not reported to surveillance using a negative binomial likelihood ratio test for continuous variables and chi-squared (or Fisher's exact test, if at least one cell count was $<5$ ) for categorical variables.

$\S$ Other races included were Black, Asian, Native Hawaiian/Other Pacific Islander, American Indian/Alaska Native, not specified, or unknown. Given the small denominator in each category, all were collapsed into a single category.

" Data are not available for all members of this cohort.

** Includes all infants with a recorded score. All scores were Finnegan or modified Finnegan.

t+ Signs and symptoms include tremors, breathing problems, blotchy skin, diarrhea, crying, fever, fussiness, gagging or retching, hiccups, hyperactive or exaggerated Moro reflex, frequent yawning, overactive reflexes, poor feeding, salivation, seizures, skin abrasions or excoriation, slow weight gain, sneezing, stuffy nose, suckling issues, sweating, vomiting, increased muscle tone, trouble sleeping, and any other symptom attributed to NAS by a clinician.

$\S \S$ Not mutually exclusive categories.

१ๆ As documented in the maternal record, infant record, or both.

*** Other opioids include codeine, fentanyl, hydrocodone, hydromorphone, kratom, morphine, and tramadol.

${ }^{++t}$ As documented in the maternal record, infant record, or both. Other substances with <20 infants exposed included alcohol, antipsychotics, barbiturates, bupropion, methamphetamine, phencyclidine, and other substances referred to directly, such as "methaqualone," or indirectly, such as "maternal polysubstance abuse."

$\S \S \S$ No infants were assigned ICD-10-CM code P96.2, "Withdrawal symptoms from therapeutic use of drugs in newborn," or P04.40, "Newborn affected by maternal anesthesia and analgesia in pregnancy, labor and delivery."
}

CSTE released the first nationally standardized NAS case definition (1) after this investigation was completed; therefore, it could not be applied to these data. Differences include that the Pennsylvania NAS case definition included prenatal opioid exposure at any time during pregnancy, and the CSTE NAS definition includes not only exposure to opioids, but also benzodiazepines and barbiturates, and limits the exposure period to $\leq 4$ weeks before delivery (1). Standardization of NAS reporting might improve with implementation of the CSTE definition.

The findings in this report are subject to at least three limitations. First, hospitals were selected to represent specific characteristics; these findings might not be representative of all hospitals in Pennsylvania or the United States. Second, in this investigation, NAS case status was determined based on infant charts alone, with maternal charts reviewed only among infants with confirmed NAS; findings might differ in investigations that can rely on both maternal and infant records to determine NAS case status. Finally, the estimate of the surveillance system's sensitivity might be biased because this investigation focused on infants who possibly had NAS and did not include chart review for a sample of all infants; this would be needed to estimate true sensitivity.

Throughout the United States, NAS surveillance is in a nascent stage; NAS surveillance can be strengthened by using a combination of diagnosis code assessment and focused medical record review. Further evaluation of NAS surveillance 
TABLE 2. Characteristics of infants with confirmed neonatal abstinence syndrome (NAS) based on medical record review ( $N=241$ ), by presence of International Classification of Diseases, Tenth Revision, Clinical Modification (ICD-CM-10) discharge diagnosis code P96.1: Neonatal withdrawal symptoms from maternal use of drugs of addiction - selected hospitals, Pennsylvania, 2018

\begin{tabular}{|c|c|c|c|}
\hline \multirow[b]{2}{*}{ Characteristic } & \multicolumn{2}{|c|}{ No.* $(\%)$ or mean (range) } & \multirow[b]{2}{*}{ p-value ${ }^{\S}$} \\
\hline & $\begin{array}{c}\text { Infants with NAS assigned } \\
\text { discharge diagnosis code P96.1 } \\
(\mathrm{N}=174)^{\dagger}\end{array}$ & $\begin{array}{l}\text { Infants with NAS not assigned } \\
\text { discharge diagnosis code P96.1 } \\
(N=67)\end{array}$ & \\
\hline \multicolumn{4}{|l|}{ Maternal race } \\
\hline White & $153(92)$ & $58(88)$ & \\
\hline Other ${ }^{\mathbb{1}}$ & $14(8)$ & $8(12)$ & 0.379 \\
\hline \multicolumn{4}{|l|}{ Maternal ethnicity } \\
\hline Hispanic or Latina & $1(1)$ & $1(2)$ & \\
\hline Not Hispanic or Latina & $160(99)$ & $63(98)$ & 0.489 \\
\hline \multicolumn{4}{|c|}{ Source of payment in maternal record } \\
\hline Medicaid & $158(95)$ & $58(88)$ & \\
\hline Private/Other & $8(5)$ & $8(12)$ & 0.048 \\
\hline Maternal age, yrs & $169^{* *} ; 29(18-41)$ & $65^{* *} ; 29(19-43)$ & 0.711 \\
\hline \multicolumn{4}{|l|}{ Infant sex } \\
\hline Male & $84(48)$ & $34(51)$ & \\
\hline Female & $90(52)$ & $33(49)$ & 0.731 \\
\hline Gestational age, wks & $169^{* * *} ; 38(33-42)$ & $66^{* *} ; 38(32-41)$ & 0.683 \\
\hline \multicolumn{4}{|l|}{ Type of hospitalization } \\
\hline Birth hospitalization & $156(90)$ & $65(97)$ & 0.109 \\
\hline Other type of admission & $17(10)$ & $2(3)$ & \\
\hline Length of stay, days & $173^{* *} ; 14(1-68)$ & $67^{* *} ; 9(2-47)$ & $<0.001$ \\
\hline \multicolumn{4}{|l|}{ NAS scores } \\
\hline Age at first NAS score, days & $168^{* *} ; 2(0-19)$ & $66^{* *} ; 1(0-6)$ & 0.031 \\
\hline First NAS Score ${ }^{\dagger \dagger}$ & $173^{* *} ; 4(0-19)$ & $66^{* *} ; 2(0-8)$ & $<0.001$ \\
\hline Age at highest NAS score, days & $166^{* *} ; 5(0-32)$ & $64^{* *} ; 3(0-10)$ & $<0.001$ \\
\hline Highest NAS score ${ }^{\dagger \dagger}$ & $173^{* *} ; 11(2-21)$ & $65^{* *} ; 9(2-16)$ & $<0.001$ \\
\hline \multicolumn{4}{|l|}{ Symptoms } \\
\hline Total number of symptoms $\$ \S$ & $173^{* *} ; 12(1-17)$ & $67^{* *} ; 9(1-16)$ & $<0.001$ \\
\hline \multicolumn{4}{|c|}{ Evidence of prenatal opioid exposure in the infant record ${ }^{\text {กิ }}$} \\
\hline Neonatal toxicology evidence & $114(66)$ & $43(64)$ & 0.845 \\
\hline Maternal toxicology evidence & $90(52)$ & $30(45)$ & 0.334 \\
\hline Maternal history & $161(93)$ & $64(96)$ & 0.567 \\
\hline \multicolumn{4}{|c|}{ Evidence of prenatal opioid exposure in the maternal prenatal or delivery record } \\
\hline Maternal toxicology evidence & $40(23)$ & $16(24)$ & 0.883 \\
\hline Maternal history & $168(97)$ & $65(97)$ & 1.000 \\
\hline \multicolumn{4}{|l|}{ Type of opioid exposure ${ }^{* * *}$} \\
\hline Buprenorphine & $116(67)$ & $44(66)$ & 0.884 \\
\hline Methadone & $53(30)$ & $15(22)$ & 0.212 \\
\hline Opiates, unspecified & $50(29)$ & $19(28)$ & 0.954 \\
\hline Heroin & $30(17)$ & $10(15)$ & 0.665 \\
\hline Oxycodone & $21(12)$ & $9(13)$ & 0.774 \\
\hline Other opioids ${ }^{\dagger+t}$ & $11(6)$ & $6(9)$ & 0.474 \\
\hline
\end{tabular}

See table footnotes on the next page.

systems after implementation of the CSTE case definition will be useful. Accurate NAS surveillance is needed to identify changes in incidence and regional differences and to plan for needed services.

Corresponding author: Elizabeth C. Ailes, EAiles@cdc.gov, 404-498-3946.

\footnotetext{
${ }^{1}$ Epidemic Intelligence Service, CDC; ${ }^{2}$ National Center on Birth Defects and Developmental Disabilities, CDC; ${ }^{3}$ Pennsylvania Department of Health; ${ }^{4}$ National Center for Immunization and Respiratory Diseases, CDC; ${ }^{5}$ Department of Research \& Evaluation, Kaiser Permanente Southern California, Pasadena, California.
}

All authors have completed and submitted the International Committee of Medical Journal Editors form for disclosure of potential conflicts of interest. No potential conflicts of interest were disclosed.

\section{References}

1. Committee on Maternal and Child Health. Neonatal abstinence syndrome standardized case definition. Atlanta, GA: Council of State and Territorial Epidemiologists; 2019. https://cdn.ymaws.com/www.cste.org/resource/ resmgr/ps/2019ps/19-MCH-01_NAS_updated_5.7.19.pdf

2. Ko JY, Patrick SW, Tong VT, Patel R, Lind JN, Barfield WD. Incidence of neonatal abstinence syndrome-28 states, 1999-2013. MMWR Morb Mortal Wkly Rep 2016;65:799-802. PMID:27513154 https://doi. org/10.15585/mmwr.mm6531a2 
TABLE 2. (Continued) Characteristics of infants with confirmed neonatal abstinence syndrome (NAS) based on medical record review ( $\mathrm{N}=241$ ), by presence of International Classification of Diseases, Tenth Revision, Clinical Modification (ICD-CM-10) discharge diagnosis code P96.1: Neonatal withdrawal symptoms from maternal use of drugs of addiction — selected hospitals, Pennsylvania, 2018

\begin{tabular}{|c|c|c|c|}
\hline \multirow[b]{2}{*}{ Characteristic } & \multicolumn{2}{|c|}{ No.* $(\%)$ or mean (range) } & \multirow[b]{2}{*}{$p$-value $e^{\S}$} \\
\hline & $\begin{array}{l}\text { Infants with NAS assigned } \\
\text { discharge diagnosis code P96.1 } \\
(\mathrm{N}=174)^{\dagger}\end{array}$ & $\begin{array}{l}\text { Infants with NAS not assigned } \\
\text { discharge diagnosis code P96.1 } \\
(\mathrm{N}=67)\end{array}$ & \\
\hline \multicolumn{4}{|l|}{ Type of other exposure ${ }^{\S \S}$} \\
\hline Tobacco & $128(74)$ & $51(76)$ & 0.684 \\
\hline Cannabis & $51(29)$ & $12(18)$ & 0.071 \\
\hline Cocaine & $28(16)$ & $10(15)$ & 0.824 \\
\hline Antidepressants & $30(17)$ & $5(7)$ & 0.054 \\
\hline Benzodiazepines & $27(16)$ & $7(10)$ & 0.311 \\
\hline Amphetamine & $23(13)$ & $4(6)$ & 0.169 \\
\hline Gabapentin & $21(12)$ & $1(1)$ & 0.011 \\
\hline \multicolumn{4}{|l|}{ Infant receipt of pharmacologic treatment for NAS } \\
\hline Yes & $105(61)$ & $2(3)$ & \\
\hline No & 66 (39) & $63(97)$ & $<0.001$ \\
\hline \multicolumn{4}{|l|}{ ICD-10-CM discharge diagnosis code } \\
\hline $\begin{array}{l}\text { P04.1, Newborn (suspected to be) affected by } \\
\text { other maternal medication }\end{array}$ & $7(4)$ & $1(1)$ & 0.449 \\
\hline $\begin{array}{l}\text { P04.2, Newborn affected by maternal } \\
\text { use of tobacco }\end{array}$ & $5(3)$ & $5(7)$ & 0.110 \\
\hline $\begin{array}{l}\text { P04.3, Newborn affected by maternal } \\
\text { use of alcohol }\end{array}$ & $0(-)$ & $1(1)$ & 0.278 \\
\hline $\begin{array}{l}\text { P04.41, Newborn affected by maternal } \\
\text { use of cocaine }\end{array}$ & $3(2)$ & $2(3)$ & 0.620 \\
\hline $\begin{array}{l}\text { P04.49, Newborn (suspected to be) affected by } \\
\text { maternal use of other drugs of addiction }\end{array}$ & $40(23)$ & $40(60)$ & $<0.001$ \\
\hline
\end{tabular}

Abbreviation: NAS = neonatal abstinence syndrome.

* Frequencies might not sum to total because of missing values.

† Includes 135 cases identified through surveillance and 39 cases identified through diagnosis code only who were assigned discharge diagnosis code P96.1.

$\S$ P-values were calculated comparing infants assigned P96.1 with infants not assigned P96.1 using a negative binomial likelihood ratio test for continuous variables and chi squared (or Fisher's exact test, if at least one cell count was $<5$ ) used for categorical variables.

" Other races included were Black, Asian, Native Hawaiian/Other Pacific Islander, American Indian/Alaska Native, not specified, or unknown. Given the small denominator in each category, all were collapsed into a single category.

** Data are not available for all members of this cohort.

${ }^{+\dagger}$ Includes all infants with a recorded score. All scores were Finnegan or modified Finnegan.

$\S \S$ Signs and symptoms include tremors, breathing problems, blotchy skin, diarrhea, crying, fever, fussiness, gagging or retching, hiccups, hyperactive or exaggerated Moro reflex, frequent yawning, overactive reflexes, poor feeding, salivation, seizures, skin abrasions or excoriation, slow weight gain, sneezing, stuffy nose, suckling issues, sweating, vomiting, increased muscle tone, trouble sleeping, and any other symptom attributed to NAS by a clinician.

१ी Not mutually exclusive categories.

*** As documented in the maternal record, infant record, or both.

t+t Other opioids include codeine, fentanyl, hydrocodone, hydromorphone, kratom, morphine, and tramadol.

$\S \S \S$ As documented in the maternal record, infant record, or both. Other substances with $<20$ infants exposed included alcohol, antipsychotics, barbiturates, bupropion, methamphetamine, phencyclidine, and other substances referred to directly, such as "methaqualone" or indirectly, such as "maternal polysubstance abuse."

ๆ ศ ก No infants were assigned ICD-10-CM code P96.2, "Withdrawal symptoms from therapeutic use of drugs in newborn," or P04.40, "Newborn affected by maternal anesthesia and analgesia in pregnancy, labor and delivery."

3. Jilani SM, Frey MT, Pepin D, et al. Evaluation of state-mandated reporting of neonatal abstinence syndrome-six states, 2013-2017. MMWR Morb Mortal Wkly Rep 2019;68:6-10. PMID:30629576 https://doi. org/10.15585/mmwr.mm6801a2

4. Lind JN, Ailes EC, Alter CC, et al. Leveraging existing birth defects surveillance infrastructure to build neonatal abstinence syndrome surveillance systems-Illinois, New Mexico, and Vermont, 2015-2016. MMWR Morb Mortal Wkly Rep 2019;68:177-80. PMID:30789880 https://doi.org/10.15585/mmwr.mm6807a3

5. Goyal S, Saunders KC, Moore CS, et al. Identification of substanceexposed newborns and neonatal abstinence syndrome using ICD-10-CM-15 hospitals, Massachusetts, 2017. MMWR Morb Mortal Wkly Rep 2020;69:951-5. PMID:32701936 https://doi.org/10.15585/ mmwr.mm6929a2
6. Bureau of Epidemiology. Neonatal abstinence syndrome: 2018 report. Harrisburg, PA: Pennsylvania Department of Health; 2019. https://www. health.pa.gov/topics/Documents/Diseases $\% 20$ and $\% 20$ Conditions/2018\%20NAS\%20REPORT.pdf

7. Maalouf FI, Cooper WO, Stratton SM, et al. Positive predictive value of administrative data for neonatal abstinence syndrome. Pediatrics 2019;143:e20174183. PMID:30514781 https://doi.org/10.1542/ peds.2017-4183

8. Elmore AL, Tanner JP, Lowry J, et al. Diagnosis codes and case definitions for neonatal abstinence syndrome. Pediatrics 2020;146:e20200567. PMID:32848030 https://doi.org/10.1542/peds.2020-0567

9. Chiang KV, Okoroh EM, Kasehagen LJ, Garcia-Saavedra LF, Ko JY. Standardization of state definitions for neonatal abstinence syndrome surveillance and the opioid crisis. Am J Public Health 2019;109:1193-7. PMID:31318590 https://doi.org/10.2105/AJPH.2019.305170 